\title{
Low-dose triptolide in combination with idarubicin induces apoptosis in AML leukemic stem-like KG1a cell line by modulation of the intrinsic and extrinsic factors
}

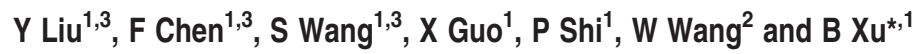

Leukemia stem cells (LSCs) are considered to be the main reason for relapse and are also regarded as a major hurdle for the success of acute myeloid leukemia chemotherapy. Thus, new drugs targeting LSCs are urgently needed. Triptolide (TPL) is cytotoxic to LSCs. Low dose of TPL enhances the cytotoxicity of idarubicin (IDA) in LSCs. In this study, the ability of TPL to induce apoptosis in leukemic stem cell (LSC)-like cells derived from acute myeloid leukemia cell line KG1a was investigated. LSC-like cells sorted from KG1a were subjected to cell cycle analysis and different treatments, and then followed by in vitro methyl thiazole tetrazolium bromide cytotoxicity assay. The effects of different drug combinations on cell viability, intracellular reactive-oxygen species (ROS) activity, colony-forming ability and apoptotic status were also examined. Combination index-isobologram analysis indicates a synergistic effect between TPL and IDA, which inhibits the colony-forming ability of LSC-like cells and induces their apoptosis. We further investigated the expression of Nrf2, HIF-1 $\alpha$ and their downstream target genes. LSC-like cells treated with both TPL and IDA have increased levels of ROS, decreased expression of Nrf2 and HIF-1 $\alpha$ pathways. Our findings indicate that the synergistic cytotoxicity of TPL and IDA in LSCs-like cells may attribute to both induction of ROS and inhibition of the Nrf2 and HIF-1 $\alpha$ pathways.

Cell Death and Disease (2013) 4, e948; doi:10.1038/cddis.2013.467; published online 5 December 2013

Subject Category: Experimental Medicine

Acute myeloid leukemia ( $A M L)$ is a lethal malignant disease. Although the induction remission rate of $\mathrm{AML}$ has been significantly improved, relapse still remains a major hurdle for successful AML chemotherapy. ${ }^{1}$ Leukemia stem cells (LSCs), first described by Lapidot et al., ${ }^{2}$ are characterized by their ability to self-renew, unlimited repopulating potential and long residence in a quiescent state of G0/G1 phase. They also express high levels of resistant-related proteins, for example, MDR1 for their multidrug-resistant characters. ${ }^{3}$ Therefore, LSCs are thought to have a central role in the relapse and refractory of AML. Consequently, new therapeutic strategies directly targeting LSCs are proposed to be critical for the ultimate curing of AML.

LSCs are usually present in small numbers, thus relatively difficult to isolate and characterize. On the other hand, some cancer cell lines are found to have cancer stem cell characteristics and therefore provide valuable tools for in vitro and in vivo studies of LSCs. KG1a is a cell line derived from a male AML patient and has LSC characteristics. KG1a cells do not spontaneously differentiate into granulocytes and macrophage-like cells and do not respond to colony-stimulating factors. ${ }^{4}$ Remarkably, even after several years in culture, many KG1a cells are still CD34 + CD38 which characterizes LSCs. ${ }^{5}$

Environment-mediated drug resistance is a transient state whereby LSCs are under protection through signals from the niche, leading to selections of secondary genetic changes with growth of cells acquiring pharmacologic resistance. ${ }^{6}$ Thus, targeting microenvironment supporting the LSCs may improve the therapeutic outcomes of AML. Previous study ${ }^{7}$ showed that the progression of leukemia in a rat model is associated with marked expansion of hypoxia, indicating the close relationship between the hypoxic microenvironment and the stemness of LSCs. Indeed, hypoxia-inducible factor-1 $\alpha(\mathrm{HIF}-1 \alpha)$, which mediates the cellular response to hypoxia, ${ }^{8}$ is upregulated in leukemia patients with poor clinical outcome. ${ }^{9}$ Suppressing the expression of HIF-1 $\alpha$ using shRNAs or small molecules in human AML LSCs, on the other hands, abrogated the colony-forming units (CFU), ${ }^{10}$ indicating the possibility of targeting HIF- $1 \alpha$ to eliminate LSCs. Mechanistically, HIF- $1 \alpha$ regulates chemokine receptor 4 (CXCR4) $)^{11}$ and $\mathrm{CXCL} 12,{ }^{12}$ both of which are essential for adhesion,

\footnotetext{
${ }^{1}$ Department of Hematology, Nanfang Hospital, Southern Medical University, Guangzhou, PR China and ${ }^{2}$ Research Institute in Healthcare Science, School of Applied Sciences, University of Wolverhampton, Wolverhampton, UK

${ }^{*}$ Corresponding author: B Xu, Department of Hematology, Nanfang Hospital, Southern Medical University, Guangzhou 510515, PR China. Tel: +86 20 61641616; Fax: +86 20 87280761; E-mail: xubingzhangjian@126.com

${ }^{3}$ These authors contributed equally to this work.

Keywords: triptolide; idarubicin; reactive-oxygen species; Nrf2; HIF-1 $\alpha$

Abbreviation: DNR, doxrubicin; ECL, enhanced chemiluminescence; $\mathrm{H}_{2} \mathrm{DCFDA}, 2^{\prime}, 7^{\prime}$-dichlorodihydrofluorescein diacetate; IDA, idarubicin; LSCs, leukemia stem cells; MTT, methyl thiazole tetrazolium bromide; PBS, phosphate-buffered saline; PVDF, polyvinylidene fluoride; TPL, triptolide; ROS, reactive-oxygen species Received 21.7.13; revised 17.10.13; accepted 21.10.13; Edited by A Finazzi-Agró
} 
migration and homing of cells. Thus, it is suggested that HIF- $1 \alpha$ facilitates recruitment, retention and survival of LSCs in a hypoxic BM microenvironment.

LSCs can also be targeted by modulating intrinsic factors. LSCs usually reside in a quiescent state, resulting in the diminished efficacy of chemotherapeutic agents targeting cycling cells. ${ }^{6}$ It has been demonstrated that oxidative stress inhibits self-renewal of LSCs. ${ }^{6}$ However, oxidation-inducing agents often trigger expression of some anti-apoptotic factors. Thus, agents simultaneously inducing oxidative stress and downregulating anti-apoptotic factors should target cancer cells more effectively. Transcription factor NF-E2-related factor 2 (Nrf2) has a vital role in activating an antioxidant response that decreases reactive-oxygen species (ROS), detoxifies harmful chemicals and ultimately protects cells from cellular damage. ${ }^{13}$ Downregulation of Nrf2 has been shown to potentially benefit cancer stem cells. ${ }^{14}$ Therefore, Nrf2 may be a promising target for elimination of LSCs.

Triptolide (TPL), a diterpenoid triepoxide, was first isolated from the medicinal plant Tripterygium wilfordii Hook $F$ (TWHF), and was structurally characterized in 1972 (Figure 1). ${ }^{15}$ Recently, TPL has also been shown to have strong anticancer effects both in vitro ${ }^{16}$ and in vivo. ${ }^{17}$ However, the clinical applications of triptolide are limited by its narrow therapeutic window and severe toxicity on the digestive, reproductive, urogenital and blood circulatory systems. ${ }^{15}$ Yet, it has been reported that a relatively low dosage of TPL enhances the cytotoxicity of some cytokines and conventional anticancer drugs indicating that TPL might be a promising chemotherapy sensitizer. ${ }^{18-20}$ Our previous study demonstrates that TPL enhances drug-sensitivity of resistant leukemia cell lines in vitro. ${ }^{21}$ Because LSC is responsible for drug resistance in conventional chemotherapy, we hypothesized that TPL may help eliminate LSCs. It has been reported that TPL has the potential of depleting quiescent CD34 + primitive CML progenitor cells, ${ }^{22}$ and that normal CD34 + hemopoietic stem cells are less sensitive to TPL than AML blasts, ${ }^{22}$ suggesting the specificity of TPL in targeting LSCs.

Idarubicin (4-demethoxydaunorubicin, IDA) is an effective drug for the early management of adult AML, ${ }^{23}$ particularly in patients expressing high level of MDR $1,{ }^{24}$ due to its ability to overcome P-glycoprotein-mediated multiple drug resistance

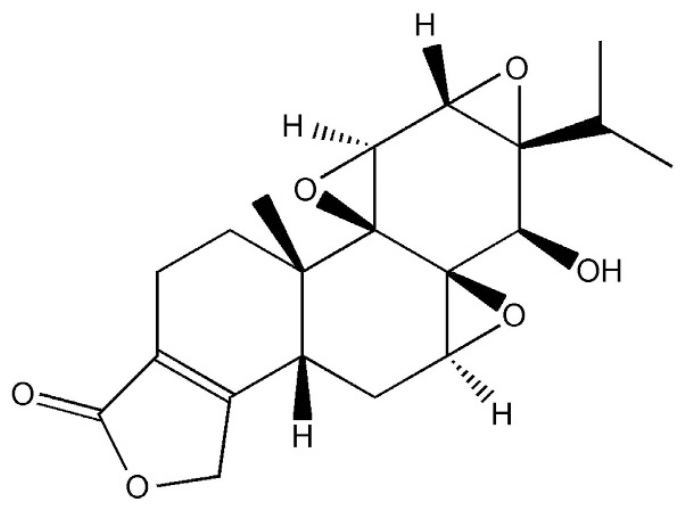

Figure 1 Structure of triptolide in vitro. ${ }^{25}$ In this study, we examined the combinational effect of TPL and IDA on KG1a leukemia stem-like cells. We also investigated the effect of IDA-TPL combination therapy on both intrinsic (oxidative stress and Nrf2) and extrinsic (hypoxia and HIF-1 $\alpha$ ) components.

\section{Results}

Collection of leukemia stem-like cells by flow cytometry sorting. CD34 + CD38 - cells in KG- $1 \alpha$ cell culture were previous found to have the characteristics of stem cells. ${ }^{5}$ Thus, fluorescence-activated cell sorting (FACS) analysis was used to sort CD34 + CD38 - cells from KG1a cell lines. As shown in Figure 2a, after sorting, $98.15 \pm 1.64 \%$ of the cells were CD34 + CD38 - , and $82.4 \pm 3.82 \%$ of these cells were in the G0/G1 phase (Figure 2b).

Enhancing effect of TPL on the cytotoxicity of IDA to leukemia stem-like cells. Cytotoxic effect of IDA or TPL to LSC-like cells was first determined by methyl thiazole tetrazolium bromide (MTT) assay. TPL is highly cytotoxic to these cells with $I C_{20}$ of $5.0 \pm 0.81 \mathrm{nM}$ and $I C_{50}$ of $20.48 \pm 1.6 \mathrm{nM}$ after $72 \mathrm{~h}$ exposure. LSC-like cells were also exposed to a series of concentrations of IDA with or without TPL $\left(\mathrm{IC}_{20} 5.0 \mathrm{nM}\right)$. As shown in Table 1 and Figure 3b, TPL significantly enhanced cytotoxicity of IDA (IC ${ }_{50-I D A}$ : $285.20 \pm 13.7 \mathrm{nM}$ versus $\mathrm{IC}_{50-\mathrm{IDA}}+\mathrm{TPL}: 27.01 \pm 0.73 \mathrm{nM}$, $P=0.000)$. Combination index was also analyzed and being presented in Figure 3c. The combination index indicates synergistic effect between IDA and TPL over a wide range of concentrations $\left(\mathrm{IC}_{50}-\mathrm{IC}_{90}\right)$. The strongest synergistic effect on LSC-like cells was detected at the lower doses of these two drugs in combination.

TPL enhanced anticancer agent IDA-induced apoptosis in LSC-like cells. To further determine the combination effect of TPL and IDA, LSC-like cells were exposed to IDA $(27 \mathrm{nM})$ with or without TPL $(5 \mathrm{nM})$ for $24 \mathrm{~h}$. The apoptotic cells were analyzed by $\mathrm{Pl} /$ Annexin $\mathrm{V}$ staining in flow cytometric analysis (Figure $3 d$; Table 2). Treatment using both IDA and TPL significantly increased the percentages of apoptotic cells compared with the treatment with IDA alone $(24.85 \pm 1.70 \%$ versus $76.87 \pm 8.34 \%, P=0.000)$.

TPL enhances the effects of IDA to inhibit colonyforming ability of LSC-like cells. Colony-forming assays were performed to explore whether TPL in combination with IDA could affect the clonogenicity of LSC-like cells. Cells were exposed to TPL $\left(\mathrm{IC}_{20}: 5.0 \mathrm{nM}\right)$ with or without IDA $(27.0 \mathrm{nM})$ for $24 \mathrm{~h}$ and then cultured in complete methylcellulose medium with recombinant cytokines for 14 days. TPL or IDA alone could reduce the colony number by slowing down the growth of the surviving cells, which causes the cell numbers in some colonies not to reach the counting threshold (40 cells). As shown in Figures $3 f$ and $g$, TPL in combination with IDA drastically reduced the colonies formed by the LSC-like cells compared with TPL alone, IDA alone and the negative control (-VE: 2097.3 $\pm 106.07 /$ well; TPL: $1387.7 \pm 40.05 /$ well; IDA: $1252 \pm 46.87 /$ well; TPL + IDA: $211 \pm 93.18 /$ well, $P=0.000)$. 

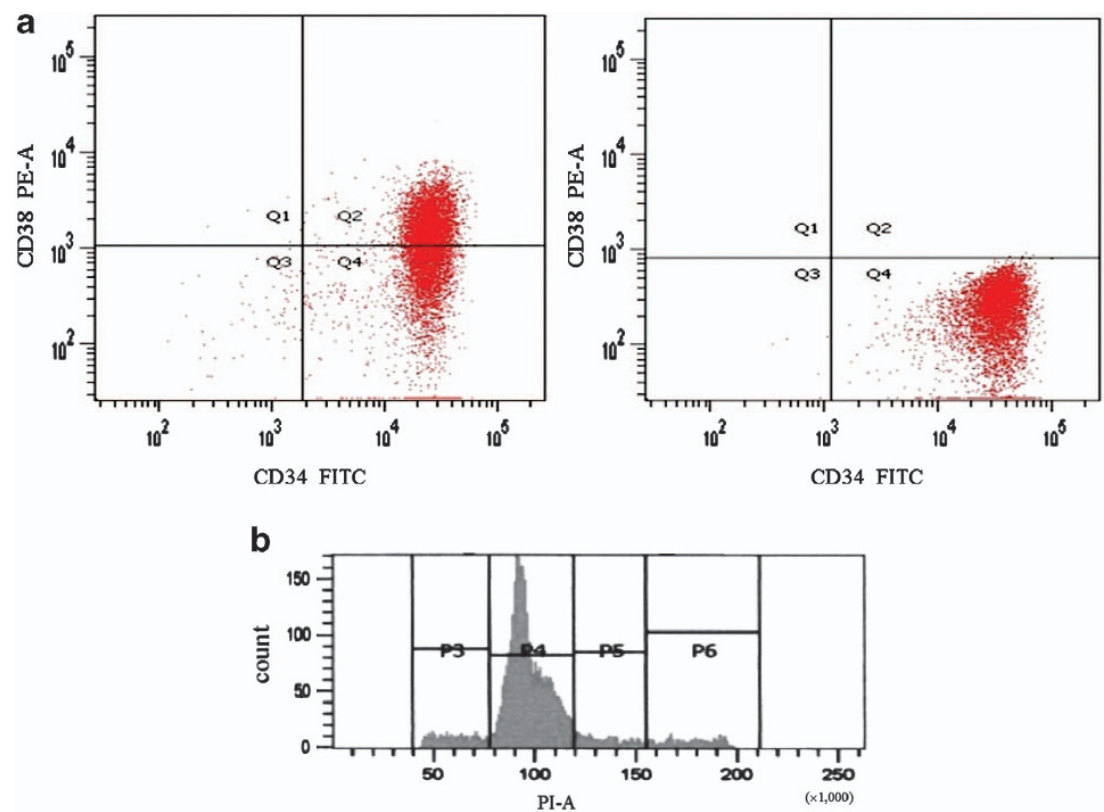

Figure 2 Leukemia stem-like cells were sorted from KG1a cell line. (a) Phenotypes of sorted cells were analyzed using flow cytometry. Before sorting, the $\mathrm{CD} 34+\mathrm{CD} 38-\mathrm{KG1a}$ cells were $53.24 \pm 2.11 \%$. After sorting, the percentage of CD34 + CD38 - cells is $98.15 \pm 1.64 \%$. (b) Cell cycle parameters in the sorted cells were analyzed with $\mathrm{G} 0 / \mathrm{G} 1$ cells being $82.4 \pm 3.82 \%$. Figures represent three independent experiments

Table 1 TPL enhances the cytotoxicity of IDA to leukemia stem and progenitor cells in vitro

\begin{tabular}{lrcc}
\hline Treatment & $\mathrm{IC}_{50}(\mathrm{nM})$ & Enhancing fold & $P$ \\
\hline IDA & $285.20 \pm 13.7$ & & \\
TPL + IDA & $27.01 \pm 0.73$ & 10.56 & 0.000 \\
\hline
\end{tabular}

Abbreviations: TPL, triptolide; IDA, idarubicin.

Leukemia stem and progenitor cells were exposed to IDA with or without TPL $\left(\mathrm{IC}_{20}, 5 \mathrm{nM}\right)$ for $72 \mathrm{~h}$ with cytotoxicity being assessed using MTT assay. Values are expressed as mean \pm S.D. of three independent experiments

TPL in combination with IDA triggers ROS activation. High ROS activity induces DNA, RNA and protein damages and leads to apoptosis in cancer cells. It has been reported that anticancer agent could induce ROS activity in cancer cells. ${ }^{18}$ To explore the role of ROS in TPL- and/or IDA-induced apoptosis, ROS levels were detected using $\mathrm{H}_{2}$ DCFDA after LSC-like cells being treated with different concentrations of TPL or IDA for $24 \mathrm{~h}$. As shown in Figure 4, ROS activation was dosage-dependent when IDA was combined with a constant concentration of TPL $\left(\mathrm{IC}_{20}\right.$ : $5 \mathrm{nM})$. The highest activity of intracellular ROS appeared in IDA 200 nM + TPL5 nM group. However, ROS activity was reduced in cells treated by higher concentrations of drugs possibly caused by cell death.

TPL in combination with IDA downregulates the Nrf2 pathway in LSC-like cells. Nrf2 is a transcription factor that activates antioxidant response and reduces ROS. ${ }^{13}$ Decrease of Nrf2 might enhance the cytotoxicity of drugs to cancer cells. A previous study has demonstrated that downregulation of Nrf2 could partially reverse chemoresistance. $^{26}$ To explore whether enhanced cytotoxicity caused by IDA and TPL combination therapy is due to the decrease in the Nrf2 expression level, Nrf2 expression was analyzed by western blotting and RT-PCR after LSC-like cells were exposed to IDA at $27 \mathrm{nM}$ plus TPL at $5 \mathrm{nM}$ for $24 \mathrm{~h}$ (Figure 5a). From Figure 5a, it was evident that in the presence of both IDA and TPL, the expression of Nrf2 was significantly reduced. Moreover, we also studied the effect of IDA + TPL on the expression levels of NQO1, GSR and $\mathrm{HO}-1$, which are downstream of Nrf2, and found that their expression levels were reduced dramatically in the presence of IDA + TPL (Figure 5a). In addition, IDA + TPL significantly reduced Nrf2 protein levels (Figure $5 \mathrm{c}$ ).

Combined use of TPL and other anticancer agents decreased HIF-1 $\alpha$ expression in LSC-like cells. Previous studies demonstrated that microenvironment where LSCs reside is vital for the survival of LSCs and thus disconnect the interactions between LSCs and their microenvironment might be a feasible way to eliminate LSCs. ${ }^{6}$ Important factors affecting microenvironment include the receptor of chemokine SDF-1 (CXCR4), the adhesion molecule VLA-4 and the tumor angiogenic transcription factor $\mathrm{HIF}-1 \alpha$. Inhibition of these factors might force LSCs to leave the niches and thus leading to the apoptosis of LSCs. We thus examined expression of CXCR4, VLA-4 and HIF-1 $\alpha$ in LSC-like cells exposed to the indicated drugs (IDA $27 \mathrm{nM}$ plus TPL $5 \mathrm{nM}$ ) for $24 \mathrm{~h}$. TPL + IDA treatment again drastically reduced HIF-1 $\alpha$ expression at both the mRNA and protein levels (Figures $5 b$ and c). Decrease of HIF- $1 \alpha$ was confirmed by downregulation of its downstream target genes such as BNIP3, vascular endothelial growth factor (VEGF) and carbonic anhydrase IX (CAIX) (Figure 5b). Similar to HIF-1 $\alpha$, TPL + IDA treatment also caused decrease of CXCR 4 and VLA-4 at the protein level. These results thus demonstrate that TPL and IDA synergistically induced apoptosis of LSC-like cells via both intrinsic and extrinsic mechanisms. 

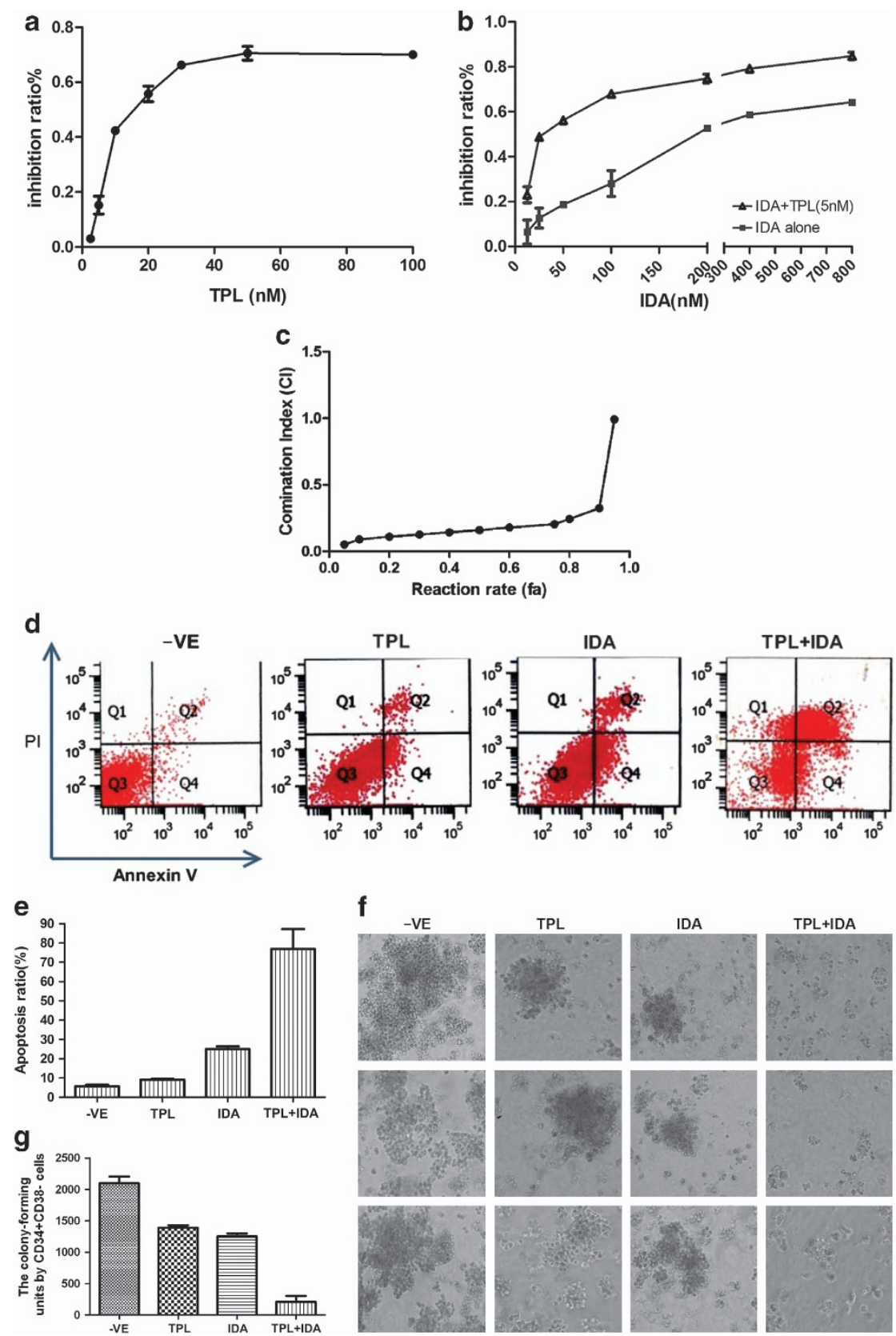

Figure 3 TPL enhances cytotoxicity of IDA to leukemia stem-like cells. (a) Leukemia stem-like cells were exposed to indicated concentrations of TPL for $72 \mathrm{~h}$ and then the viability was determined by MTT assay. (b) Cytotoxicity of IDA was determined after exposure to indicated concentrations of IDA with or without TPL (IC ${ }_{20}, 5 \mathrm{nM}$ ). (c) Synergy was determined by combination index $(\mathrm{Cl})$ analysis and expressed as $\mathrm{Cl}$ versus fraction affected. (d) Leukemia stem-like cells were exposed to different treatments (IDA: $27 \mathrm{nM}$, TPL: $5 \mathrm{nM}$ ) with apoptotic ratio detected using flow cytometry. (e) Histogram of apoptotic ratio in leukemia stem-like cells. (f) Colonies were formed from leukemia stem-like cells being exposed to IDA ( $27 \mathrm{nM}$ ) with or without TPL $(5 \mathrm{nM})$ for $24 \mathrm{~h}$. (g) The colony-forming units grown by leukemia stem-like cells were counted (40 or more cells per unit) per well. All treated groups have decreased colony units with the largest scale of decrease occurring in combined group. TPL, triptolide; IDA, idarubicin; values are expressed as mean \pm S.D. of three independent experiments

\section{Discussion}

In recent years, cumulating evidence demonstrates that TPL possesses anticancer properties by its marvelous antiproliferative activity and induction of apoptosis in a variety of cancer types in vitro and in vivo. ${ }^{16,18-20}$ However, use of TPL alone for cancer is limited by its non-specific toxicity and low therapeutic index. ${ }^{15}$ Nevertheless, numerous studies have reported the potential of using TPL to reverse chemoresistance in some leukemia cell lines. ${ }^{16,27,28}$ Our previous study has also demonstrated the ability of TPL to enhance the drug-sensitivity in resistant myeloid leukemia cell lines. ${ }^{21}$ LSCs have already been demonstrated to be closely related to chemo-resistance. ${ }^{6}$ Thus, we are interested to know whether TPL could enhance the ability of conventional antileukemia drugs to eliminate LSCs. However, it is not feasible to isolate 
Table 2 TPL enhances the apoptosis induced by IDA in leukemia stem and progenitor cells in vitro

\begin{tabular}{lcccc}
\hline$-V E$ & TPL & IDA & IDA + TPL & $P$ \\
\hline $5.63 \pm 0.67 \%$ & $9.11 \pm 0.33 \%$ & $24.85 \pm 1.70 \%$ & $76.87 \pm 8.34 \%{ }^{*}$ & 0.000
\end{tabular}

Abbreviations: TPL, triptolide; IDA, idarubicin

Leukemia stem and progenitor cells were exposed to different treatment for $24 \mathrm{~h}$ (IDA: $27 \mathrm{nM}$, TPL: $5 \mathrm{nM}$ ). The apoptotic ratios were detected using flow cytometry. "versus -VE, $P=0.000$ \#versus IDA, $P=0.000$. Values are expressed as mean \pm S.D. of three independent experiments

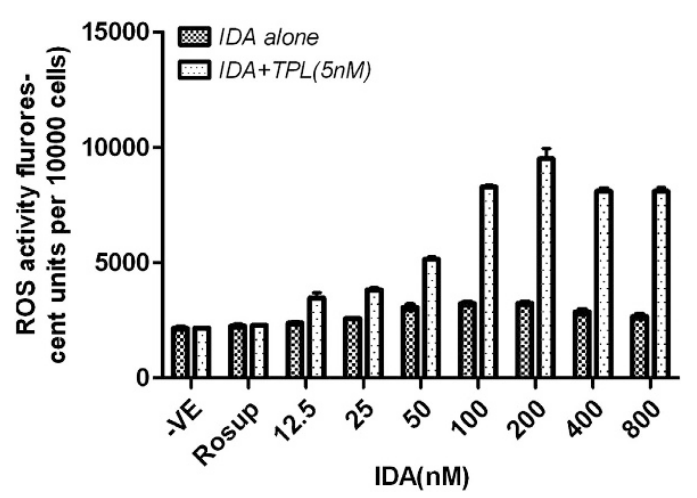

Figure 4 TPL in combination with IDA generates ROS in leukemia stem-like cells. Leukemia stem-like cells were incubated with indicated concentrations of IDA with or without TPL $(5 \mathrm{nM})$ for $24 \mathrm{~h}$. The DCF fluorescence was detected as described in Methods. TPL intensively synergized IDA in inducing ROS activity in leukemia stem-like cells in a concentration-dependent manner. TPL, triptolide; IDA, idarubicin; values are expressed as mean \pm S.D. of three independent
LSCs from fresh bone marrow. Therefore, we used stem-like cells isolated from KG1a cell lines to perform our experiments. Our previous study has demonstrated the superiority of IDA compared with doxorubicin (DNR) in AML patients with high expression of MDR $1,{ }^{24}$ indicating that IDA might be superior to DNR in elimination of LSCs.

In this study, we demonstrated that low concentrations of TPL can enhance IDA-induced apoptosis in leukemia stemlike cells through modulating both intrinsic and extrinsic components (Figure 6). TPL was highly toxic to LSC-like cells in a dosage-dependent manner (Figure 3a). Elimination of LSC-like cells required the treatment of IDA at a relatively high concentration $\left(\mathrm{IC}_{50}: 285.20 \pm 13.7 \mathrm{~nm}\right)$. However, low concentration of TPL $\left(\mathrm{IC}_{20}: 5 \mathrm{nM}\right)$ significantly enhanced the cytotoxicity of IDA in LSC-like cells by 10 -fold (Table 1; Figure $3 b$ ). Moreover, $\mathrm{Cl}$ isobologram analysis showed that TPL and IDA had synergistic effect when fraction affected was below $90 \%$ (Figure 3c). Our results also indicate that TPL enhanced IDA-induced apoptosis in LSC-like cells (Table 2; Figure 3d). In comparison with IDA alone treated cells, after $24 \mathrm{~h}$ exposure to IDA and TPL, the apoptotic cell population was significantly increased in leukemia stem-like cells ( $24.85 \pm 1.70 \%$ versus $76.87 \pm 8.34 \%, P=0.000$ ). Moreover, as shown in Figure $3 \mathrm{f}$, clonogenicity of leukemia stem-like cells was also inhibited by TPL + IDA treatment.

We further investigated the mechanism of the cytotoxicity of TPL in LSC-like cells. Abnormal hypoxic microenvironment influences the survival of LSCs, whereas HIF- $1 \alpha$ mediates the cellular response to hypoxia. ${ }^{6} \mathrm{HIF}-1 \alpha$ can induce the expression of multiple target genes such as VEGF ${ }^{29}$ (an important angiogenesis factor), CXCR $4^{30}$ (an important factor regulating cell migration), adenovirus E1B 19-kDa-interacting protein
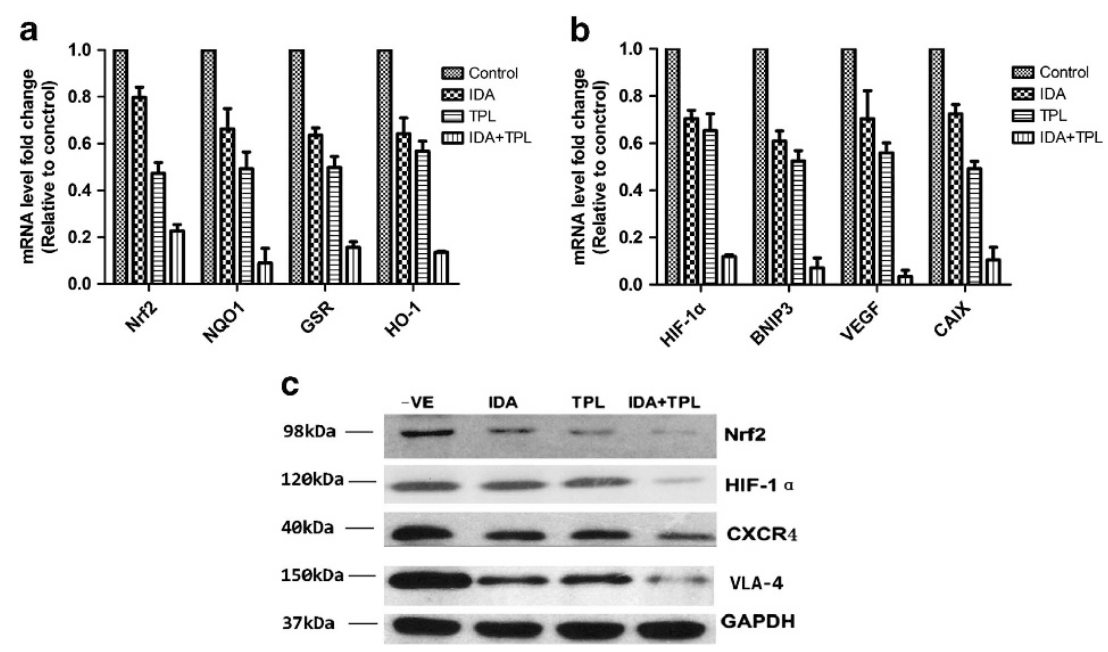

Figure 5 TPL in combination of IDA decreases the expression of Nrf2, HIF-1 $\alpha$ and its downstream genes. (a) Nrf2 and its downstream genes were assessed using RT-PCR after leukemia stem-like cells being exposed to indicated drugs (IDA, $27 \mathrm{nM}$ plus TPL, $5 \mathrm{nM}$ ) for $24 \mathrm{~h}$. RT-PCR result showed that the expression of Nrf2 and NQ01, GSR and HO-1 genes decreased in single-drug-treated groups while the largest scale of decrease occurred in leukemia stem-like cells exposed to IDA plus TPL. (b) HIF-1 $\alpha$ and its downstream genes were assessed using RT-PCR after leukemia stem-like cells being exposed to indicated drugs (IDA, $27 \mathrm{nM}$ plus TPL, $5 \mathrm{nM}$ ) for $24 \mathrm{~h}$. RT-PCR result shows that the expression of HIF-1 $\alpha$ and BNIP3, VEGF and CAIX genes decreased in single-drug-treated groups while the largest scale of decrease occurred in leukemia stem-like cells exposed to IDA plus TPL. (c) Western blotting analysis of the expression of Nrf2, HIF-1 $\alpha$, CXCR4 and VLA-4 in the leukemia stem-like cells treated as indicated. Nrf2, HIF-1 $\alpha$, CXCR4 and VLA-4 were assessed using western blotting analysis after leukemia stem-like cells being exposed to indicated drugs (IDA, $27 \mathrm{nM}$ plus TPL, $5 \mathrm{nM}$ ) for $24 \mathrm{~h}$. The expression of Nrf2, HIF-1 $\alpha$, CXCR4 and VLA-4 decreased in treated groups especially in cells treated by IDA + TPL group. GAPDH was used as a loading control. This figure represents three independent experiments. TPL: triptolide; IDA: idarubicin; values are expressed as mean \pm S.D. of three independent experiments 
3 (BNIP3) $^{31}$ (a pro-cell death member of the Bcl-2 family) and $\mathrm{CAIX}^{31}$ (a marker for hypoxia). Thus, HIF-1 $\alpha$ can activate downstream pathways involved in proliferation, metabolism, differentiation and angiogenesis, ${ }^{32,33}$ which likely account for the ability of HIF- $1 \alpha$ to keep leukemic blasts at undifferentiated stages and therefore maintain a larger number of LSCs in the bone marrow. ${ }^{34}$ It has been shown that silencing HIF-1 $\alpha$ in Jurkat cells significantly decreased their migration and infiltration capacity. ${ }^{35}$ In this study, TPL combined with IDA reduced both protein and $\mathrm{mRNA}$ levels of HIF-1 $\alpha$ in LSC-like cells (Figures $5 \mathrm{~b}$ and $\mathrm{c}$ ). In addition, the expression levels of HIF-1 $\alpha$ downstream genes, such as BNIP3, VEGF and CAIX, were also reduced (Figure $5 b$ ). The downregulation of VLA-4 and CXCR4 by TPL + IDA were also observed at the protein level (Figure 5c). CXCR4 is an important chemokines for cell adhesion and expression of VLA-4 to keep LSCs in the niche. ${ }^{36}$ Previous study shows a neutralizing VLA-4 antibody, in conjunction with cytarabine, prevented the development of $\mathrm{AML}$ in a xenograftmodel, supporting the role of VLA-4 in the sensitivity of AML cells to chemotherapy, ${ }^{37}$ whereas targeting CXCR4 with small-molecule pharmacologic inhibitors has been shown to be efficacious in preclinical models of AML. ${ }^{38}$ Taken these data together, TPL in combination with IDA could significantly influence the extrinsic components, for example, $\mathrm{HIF}-1 \alpha$ and its adhesion molecules and chemokines, for example, CXCR4 and VLA-4.

Intrinsic components are also vital factors that affect the survival of LSCs. ${ }^{6}$ ROS is involved in essentially every stage of cancer development. ${ }^{39}$ Although cancer cells always possess higher levels of ROS than normal cells, ${ }^{39}$ LSCs contain relatively lower levels of ROS than non-stem cancer cells. ${ }^{40}$ Thus, many anticancer agents, for example, Niclosmide, ${ }^{41}$ Parthenolide ${ }^{42}$ kill LSCs partially through activation of ROS. In our study, ROS elevation induced by TPL and IDA was also observed in a concentration-dependent manner (Figure 4).

Agents that induce oxidative stress alone might not be efficient enough to eliminate LSCs, as the induction of protective factors. Nrf2, induced by ROS, is a transcription factor that has a vital role in activating an antioxidant

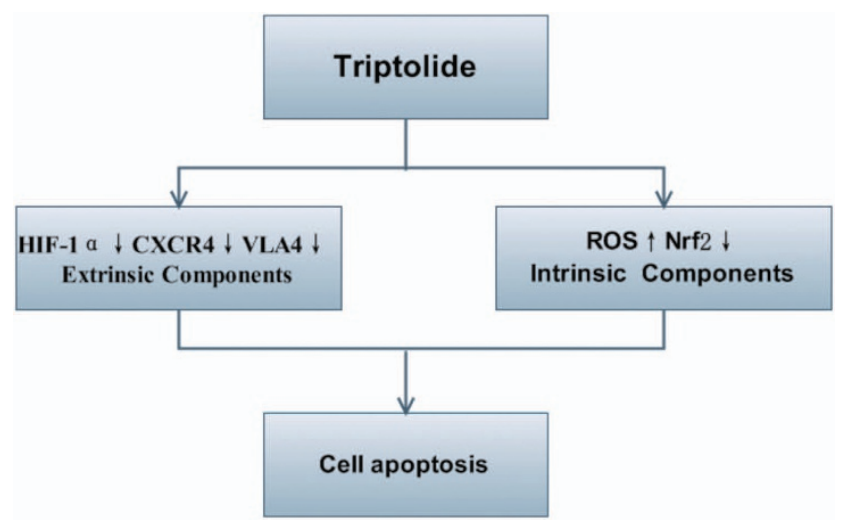

Figure 6 Mechanism of TPL-induced apoptosis in leukemia stem-like cells. A relatively low concentration of TPL in combination with IDA could induce apoptosis of leukemia stem-like cells in vitro through influence of extrinsic components, for example, downregulation of HIF- $1 \alpha$, CXCR 4 and VLA-4 as well as intrinsic components, for example, increase of ROS level and decrease of Nrf2 response, which decreases the ROS level, detoxifies harmful chemicals and eventually protects against cellular damage. ${ }^{43}$ It is known that high basal nuclear levels of Nrf2 in leukemia cells could reduce their sensitivity to proteasome inhibitors. ${ }^{44}$ Moreover, stabilization of Nrf2 might contribute to the induction of cancer stem cells. ${ }^{14}$ Thus, Nrf2 might be a potent target for elimination of LSCs. We found that TPL alone or in combination with IDA reduced mRNA and protein levels of Nrf2 in LSC-like cells (Figures $5 a$ and $c$ ) as well as downstream genes of Nrf2 such as NAD(P)H, NQO1, GSR and $\mathrm{HO}-1 .{ }^{13}$ These target genes of Nrf2 can form a network of reactions, resulting in protection against oxidative insults and enhancing cell survival. ${ }^{13}$ Downregulation of Nrf2 and its target genes therefore might overcome adaptive Nrf2 protection for LSC-like cells, leading to cell apoptosis.

In conclusion, our data clearly demonstrate that a relatively low concentration of TPL in combination with IDA-induced apoptosis of LSC-like cells by affecting both extrinsic and intrinsic factors. TPL and IDA downregulated extrinsic factors HIF- $1 \alpha$, CXCR4 and VLA-4 as well as intrinsic components, for example, increase of the ROS level and decrease of Nrf2 (Figure 6). The ability of combination of TPL and IDA to eliminate LSC-like cells in vitro provides a basis for using them in treating relapse and refractory leukemia patients.

\section{Materials and Methods}

Chemicals and reagents. Triptolide (MW: 360.40 , Sigma, Dorset, UK) was dissolved in DMSO as a $100 \mu \mathrm{M}$ stock solution and freshly diluted in culture medium before use. Idarubicin (Pfizer Japan Inc., Tokyo, Japan) was dissolved in phosphate-buffered saline (PBS) as a $1 \mathrm{mM}$ stock solution at $-20^{\circ} \mathrm{C}$.

Cell culture and sorting. The human AML cell line KG1a was maintained in suspension culture with Iscove's Modified Dulbecco's medium (IMDM, Gibco BRL, Rockville, MD, USA) medium supplemented with $10 \%$ fetal bovine serum (FBS; Gibco-BRL) at $37^{\circ} \mathrm{C}$ in a water-saturated atmosphere with $5 \% \mathrm{CO}_{2} . \mathrm{CD} 34+$ CD38 - leukemia stem-like cells were enriched by indirectly labeling with CD34FITC, CD38-PE and anti-FITC Microbeads according to the manufacturer's instructions (Becton Dickinson Biosciences, Oxford, UK). During the sorting procedure, cells were kept at $4^{\circ} \mathrm{C}$. Briefly, cell suspensions were centrifuged at $300 \times g$ for $10 \mathrm{~min}$ and washed in PBS buffer. Then, cell pellets were resuspended in PBS buffer containing $0.5 \%$ bovine serum albumin (BSA), CD34-FITC and CD38-PE antibodies were added, and the cells were incubated for $30 \mathrm{~min}$. After washing, cell pellets were resuspended in buffer with anti-FITC microbeads, and the cells were incubated for $15 \mathrm{~min}$. After washing, CD34-positive and CD38-negative cells were depleted by FACS analysis (FACS Caliber; Becton Dickinson Biosciences).

Immunophenotype. The cell surface immunophenotype of CD34+ CD38 - leukemia stem-like cells sorted by KG1a cell line was characterized with a series of murine monoclonal antibodies (CD38-FITC, CD34 + FITC, $\mathrm{BD}$ Biosciences). Binding of antibodies was assessed by flow cytometry (Becton Dickinson, FACS 440).

Cell cycle analysis. DNA content in CD34 + CD38 - cells was determined by flow cytometry analysis. $1 \times 10^{6}$ cells were harvested and washed with PBS, resuspended and fixed by slow addition of $2 \mathrm{ml} 100 \%$ ice-cold methanol on mixer and stored at $-20^{\circ} \mathrm{C}$ until analysis. Following fixation, the cells were washed with cold PBS, then stained with propidium iodide (PI, KeyGen Biotech, Nanjing, China) in sodium citrate $(1 \mathrm{mg} / \mathrm{ml}$; KeyGen Biotech) containing $100 \mu \mathrm{g} / \mathrm{ml}$ RNase A (KeyGen Biotech), $20 \mu \mathrm{g} / \mathrm{ml} \mathrm{PI}$ and $0.1 \%$ Triton X-100 (KeyGen Biotech) for $30 \mathrm{~min}$ at $37^{\circ} \mathrm{C}$ temperature. PI fluorescence was analyzed by FACS analysis (FACS Caliber; Becton Dickinson Biosciences). Cell cycle distributions were calculated with Cell Quest (BD Biosciences) software. 
MTT assay. Cytotoxicity of TPL and IDA was determined in sorted leukemia stem-like cells using the MTT assay. Cells were dispensed in 96-well tissue culture plates (Costar, Cambridge, MA, USA; $1 \times 10^{5}$ per well) in TPL, IDA or TPL + IDA containing medium for $72 \mathrm{~h}$ at escalating concentrations in the presence or absence of each drug, under the condition of $37^{\circ} \mathrm{C}$ in humidified $5 \% \mathrm{CO}_{2}$. MTT $(5 \mathrm{mg} / \mathrm{ml})$ in PBS was added to each well and the plate was incubated for $4 \mathrm{~h}$. Formazan crystals were dissolved in $200 \mu \mathrm{l}$ DMSO and the optical density (OD) of wells was measured after $15^{\prime}$ with a Multisckan MCC340 microplate reader (Labsystem, Helsinki, Finland) at $490 \mathrm{~nm}$. All experiments were in triplicate. The $\mathrm{IC}_{50}$ (the half maximal inhibitory concentration) was determined by Software (Biosoft, Cambridge, UK) following the viability results, the cytotoxicity combination index $(\mathrm{Cl} \%)$ was calculated by the equation $(1-\mathrm{OD}$ treated well OD untreated well $) \times 100 \%$.

Flow cytometric analysis of apoptosis. Apoptotic status was also assessed using an Annexin V staining kit (IQ products) following the manufacturer's instruction. Briefly, after exposure to TPL, IDA or TPL + IDA containing medium for $24 \mathrm{~h}$ in the six-well plates $\left(1 \times 10^{6}\right.$ per well), cells were harvested, washed with PBS twice and resuspended in $500 \mu$ l-binding buffer containing both $5 \mu \mathrm{l}$ Annexin V-FITC and PI (Sigma-Aldrich, St. Louis, MO, USA) and were incubated for $30 \mathrm{~min}$ at $4{ }^{\circ} \mathrm{C}$ in the dark. The contents in 10000 cells from each sample binding of FITC-conjugated Annexin V and PI was then measured by FACS analysis using FACS Caliber (Becton Dickinson Biosciences) and the data were analyzed using Cell Quest (BD Biosciences) software.

Colony-forming assay. The colony-forming assay was performed as described previously. ${ }^{45}$ Leukemia stem-like cells were collected from six-well plates (Costar), which were cultured in TPL, IDA or TPL + IDA containing medium for $24 \mathrm{~h}\left(1 \times 10^{6}\right.$ per well). Then $1 \times 10^{4} \mathrm{CD} 34+\mathrm{CD} 38-$ cells were plated for colony formation assay for 14 days in complete methylcellulose medium with recombinant cytokines (MethoCult GF $+\mathrm{H} 4434$; Stem Cell Technology, Vancouver, BC, Canada). CFU in culture (CFU-C) were performed in triplicate and were subsequently scored with an inverted microscope (Olympus, Tokyo, Japan). Colonies were defined as clusters consisting of 40 or more cells.

Intracellular ROS measurement. The intracellular ROS levels were determined by $2^{\prime}, 7^{\prime}$-dichlorodihydrofluorescein diacetate $\left(\mathrm{H}_{2} \mathrm{DCFDA}\right)$ probe (Invitrogen, Paisley, UK) as described previously. Briefly, the leukemia stem-like cells $\left(1 \times 10^{6}\right.$ per well) were cultured in six-well plate with the treatments of TPL, IDA or TPL + IDA containing medium at escalating concentrations for $24 \mathrm{~h}$. Rosup were used ( $1 \mu \mathrm{l}$ in $50 \mathrm{mg} / \mathrm{ml}, 24 \mathrm{~h}$ ) as a positive control for ROS production. The cells were harvested and washed in PBS buffer twice, incubated in $1 \mathrm{ml}$ of serum-free IMDM medium (Hyclone, Beijing, China) containing $10 \mu \mathrm{M}$ of $\mathrm{H}_{2}$ DCFDA for $30 \mathrm{~min}$ at $37^{\circ} \mathrm{C}$. The cells were harvested, washed in serum-free IMDM medium buffer twice to remove the remaining $\mathrm{H}_{2}$ DCFDA. After that, the fluorescence in the supernatant was measured at excitation $490 \mathrm{~nm}$ and emission $520 \mathrm{~nm}$ using FACS analysis (FACS Caliber; Becton Dickinson Biosciences). The data were presented as fluorescent units $/ 1 \times 10^{4}$ cells.

Western blot analysis. The cytoplasmic protein ( $50 \mu \mathrm{g} / \mathrm{lane}$ ) from each sample was dissolved in $10 \%$ SDS-polyacrylamide gel electrophoresis (PAGE), transferred to a polyvinylidene fluoride (PVDF) membrane (Millipore, Billerica, MA USA) and blotted with the various antibodies. Non-specific binding was avoided by blocking the PVDF membrane with 5\% skimmed milk in TBS-T for $1 \mathrm{~h}$. The $5 \%$ skimmed milk in TBS-T was also used to dilute primary (HIF-1 $\alpha$, rabbit polyclonal, 1:1000, MP; CXCR4, rabbit polyclonal 1:1000, MP; Nrf2, rabbit polyclonal, $1: 1000$, CST) and HRP-conjugated monoclonal secondary (1:5000; Amersham Pharmacia Biotech, Buckinghamshire, UK) antibodies. The membranes were incubated with the primary antibodies overnight at $4{ }^{\circ} \mathrm{C}$ and in the secondary antibody for $1 \mathrm{~h}$ at room temperature. The quantity of protein loaded was verified by staining the same membranes with GAPDH antibody (1:2000, Sigma-Aldrich, Dorset, UK). The signals were detected on X-ray films using an enhanced chemiluminescence $(E C L)$ western blotting detection kit (Amersham Pharmacia Biotech).

Quantitative real-time PCR. Cells were lysed with the Trizol reagent and total RNA was isolated with chloroform and isopropyl alcohol. One microgram RNA was subjected to reverse transcription with the RT reagent kit (TaKaRa, Dalian, China) according to the manufacturer's instructions. Then the cDNA was amplified by real-time PCR with the SYBR Prime Script RT-PCR kit (Takara) with the following primers (synthesized by Takara): $\beta$-actin, $5^{\prime}$-TGACGGGGTCACCCACA CTGTGCCCATCTA-3' (forward), 5'-CTAGAAGCATTGCGGTCGACGATGGAGG G-3' (reverse); HIF-1 $\alpha, 5^{\prime}$-CTCAAAGTCGGACAGCCTCA-3' (forward), $5^{\prime}$-CCCTG CAGTAGGTTTCTGCT-3' (reverse); CAIX,5'-CTTGGAAGAAATCGCTGAGG-3' (forward), 5'-TGGAAGTAGCGGCTGAAGTC-3' (reverse); BNIP3, 5'-TGCTGCTC TCTCATTTGCTG-3' (forward), 5'-GACTCCAGTTCTTCATCAAAAGGT-3' (reverse); VEGF, 5'-CTACCTCCACCATGCCAAGT-3' (forward), 5'-CCACTTCGT GATGATTCTGC-3' (reverse). HO-1, 5'-TTGCCAGTGCCACCAAGTTC-3' (forward); 5'-TCAGCAGCTCCTGCAACTCC-3' (reverse). GSR, 5'-CCTGATCGCCAC AGGTGGTA-3' (forward); 5'-CTGCCATCTCCACAGCAATGTAA-3' (reverse); NQ01, 5'-GTGGCAGTGGCTCCATGTACTC-3' (forward); 5'-GAGTGTGCCCAAT GCTATATGTCAG-3' (reverse); Nrf2, 5'-TGGGCCCATTGATGTTTCTG-3' (forward); $5^{\prime}$-TGCCACACTGGGACTTGTGTTTA-3' (reverse). The alteration of mRNA expression in cells treated with or without triptolide was assessed by the $2^{-\Delta \Delta} \mathrm{Ct}$ method.

Statistical analysis. SPSS 13.0 Student's t-test and one way analysis of variance (ANOVA) followed by the Tukey's Multiple Comparison test were used to calculate the differences between the results. Data were expressed as mean \pm S.D., and all the $P$-values were given for two-sided tests and $P \leq 0.05$ was considered as significantly changes.

\section{Conflict of Interest}

The authors declare no conflict of interest.

Acknowledgements. This work was financially supported by National Nature Science Foundation of China, PR China (No. 81070425) and the technology program of Guangdong Province, PR China (No. 2011B031800063).

1. Damiani D, Tiribelli M, Raspadori D, Michelutti A, Gozzetti A, Calistri E et al. The role of MDR-related proteins in the prognosis of adult acute myeloid leukaemia (AML) with normal karyotype. Hematol Oncol 2007; 25: 38-43.

2. Lapidot T, Grunberger T, Vormoor J, Estrov Z, Kollet O, Bunin N et al. Identification of human juvenile chronic myelogenous leukemia stem cells capable of initiating the disease in primary and secondary SCID mice. Blood 1996; 88: 2655-2664.

3. de Figueiredo-Pontes LL, Pintao MC, Oliveira LC, Dalmazzo LF, Jacomo RH, Garcia AB et al. Determination of P-glycoprotein, MDR-related protein 1, breast cancer resistance protein, and lung-resistance protein expression in leukemic stem cells of acute myeloid leukemia. Cytometry B Clin Cytom 2008; 74: 163-168.

4. Fardel O, Payen L, Courtois A, Drenou B, Fauchet R, Rault B. Differential expression and activity of P-glycoprotein and multidrug resistance-associated protein in CD34-positive KG1a leukemic cells. Int J Oncol 1998; 12: 315-319.

5. She M, Niu X, Chen X, Li J, Zhou M, He Y et al. Resistance of leukemic stem-like cells in AML cell line KG1a to natural killer cell-mediated cytotoxicity. Cancer Letters 2012; 318: 173-179

6. Konopleva MY, Jordan CT. Leukemia stem cells and microenvironment: biology and therapeutic targeting. J Clin Oncol 2011; 29: 591-599.

7. Mortensen BT, Jensen PO, Helledie N, Iversen PO, Ralfkiaer E, Larsen JK et al. Changing bone marrow micro-environment during development of acute myeloid leukaemia in rats. Br J Haematol 1998; 102: 458-464

8. Marin-Hernandez A, Gallardo-Perez JC, Ralph SJ, Rodriguez-Enriquez S, Moreno-Sanchez R. HIF-1alpha modulates energy metabolism in cancer cells by inducing over-expression of specific glycolytic isoforms. Mini Rev Med Chem 2009; 9: 1084-1101.

9. Deeb G, Vaughan MM, McInnis I, Ford LA, Sait SNJ, Starostik P et al. Hypoxia-inducible factor- $1 \alpha$ protein expression is associated with poor survival in normal karyotype adult acute myeloid leukemia. Leuk Res 2011; 35: 579-584.

10. Wang Y, Liu Y, Malek SN, Zheng P. Targeting HIF1alpha eliminates cancer stem cells in hematological malignancies. Cell Stem Cell 2011; 8: 399-411.

11. Staller P, Sulitkova J, Lisztwan J, Moch H, Oakeley EJ, Krek W. Chemokine receptor CXCR4 downregulated by von Hippel-Lindau tumour suppressor pVHL. Nature 2003; 425: 307-311.

12. Ceradini DJ, Kulkarni AR, Callaghan MJ, Tepper OM, Bastidas N, Kleinman ME et al. Progenitor cell trafficking is regulated by hypoxic gradients through HIF-1 induction of SDF-1. Nat Med 2004; 10: 858-864.

13. Sporn MB, Liby KT. NRF2 and cancer: the good, the bad and the importance of context. Nature Rev Cancer 2012; 12: 564-571.

14. Achuthan S, Santhoshkumar TR, Prabhakar J, Nair SA, Pillai MR. Drug-induced senescence generates chemoresistant stemlike cells with low reactive oxygen species. J Biol Chem 2011; 286: 37813-37829. 
15. Zhou ZL, Yang YX, Ding J, Li YC, Miao ZH. Triptolide: structural modifications, structureactivity relationships, bioactivities, clinical development and mechanisms. Nat Prod Rep 2012; 29: 457-475.

16. Chan EW, Cheng SC, Sin FW, Xie Y. Triptolide induced cytotoxic effects on human promyelocytic leukemia, T cell lymphoma and human hepatocellular carcinoma cell lines. Toxicol Lett 2001; 122: 81-87.

17. Zhao $H$, Yang $Z$, Wang $X$, Zhang $X$, Wang $M$, Wang $Y$ et al. Triptolide inhibits ovarian cancer cell invasion by repression of matrix metalloproteinase 7 and 19 and upregulation of E-cadherin. Exp Mol Med 2012; 44: 633-641.

18. Xu B, Guo X, Mathew S, Armesilla AL, Cassidy J, Darling JL et al. Triptolide simultaneously induces reactive oxygen species, inhibits NF-kappaB activity and sensitizes 5 -fluorouracil in colorectal cancer cell lines. Cancer Lett 2010; 291: 200-208.

19. Ding X, Zhou X, Zhang H, Qing J, Qiang H, Zhou G. Triptolide augments the effects of 5-lipoxygenase RNA interference in suppressing pancreatic tumor growth in a xenograft mouse model. Cancer Chemother Pharmacol 2012; 69: 253-261.

20. Huang $X$, Yang M, Jin J. Triptolide enhances the sensitivity of multiple myeloma cells to dexamethasone via microRNAs. Leuk Lymphoma 2012; 53: 1-8.

21. Chen F, Liu Y, Wang S, Guo X, Shi P, Wang W et al. Triptolide, a Chinese herbal extract, enhances drug sensitivity of resistant myeloid leukemia cell lines through downregulation of HIF-1alpha and Nrf2. Pharmacogenomics 2013; 14: 1305-1317.

22. Mak DH, Schober WD, Chen W, Konopleva M, Cortes J, Kantariian HM et al. Triptolide induces cell death independent of cellular responses to imatinib in blast crisis chronic myelogenous leukemia cells including quiescent CD34 + primitive progenitor cells. Mol Cancer Ther 2009; 8: 2509-2516.

23. Berman E, Heller G, Santorsa J, McKenzie S, Gee T, Kempin S et al. Results of a randomized trial comparing idarubicin and cytosine arabinoside with daunorubicin and cytosine arabinoside in adult patients with newly diagnosed acute myelogenous leukemia. Blood 1991; 77: 1666-1674.

24. Shi $P$, Zha J, Guo X, Chen F, Fan Z, Huang F et al. Idarubicin is superior to daunorubicin in remission induction of de novo acute myeloid leukemia patients with high MDR1 expression. Pharmacogenomics 2013; 14: 17-23.

25. Fukushima $\mathrm{T}$, Inoue $\mathrm{H}$, Takemura $\mathrm{H}$, Kishi $\mathrm{S}$, Yamauchi $\mathrm{T}$, Inai $\mathrm{K}$ et al. Idarubicin and idarubicinol are less affected by topoisomerase II-related multidrug resistance than is daunorubicin. Leuk Res 1998; 22: 625-629.

26. Tang X, Wang H, Fan L, Wu X, Xin A, Ren $\mathrm{H}$ et al. Luteolin inhibits Nrf2 leading to negative regulation of the Nrf2/ARE pathway and sensitization of human lung carcinoma A549 cells to therapeutic drugs. Free Radic Biol Med 2011; 50: 1599-1609.

27. Carter BZ, Mak DH, Schober WD, Dietrich MF, Pinilla C, Vassilev LT et al. Triptolide sensitizes AML cells to TRAIL-induced apoptosis via decrease of XIAP and p53-mediated increase of DR5. Blood 2008; 111: 3742-3750.

28. Pigneux A, Mahon FX, Uhalde M, Jeanneteau M, Lacombe F, Milpied N et al. Triptolide cooperates with chemotherapy to induce apoptosis in acute myeloid leukemia cells Exp Hematol 2008; 36: 1648-1659.

29. Ioannou M, Pyrpasopoulou A, Simos G, Paraskeva E, Nikolaidou C, Venizelos I et al. Upregulation of VEGF expression is associated with accumulation of HIF-1alpha in the skin of naive scleroderma patients. Mod Rheumatol 2013; 23: 1245-1248.

30. Oh YS, Kim HY, Song IC, Yun HJ, Jo DY, Kim S et al. Hypoxia induces CXCR4 expression and biological activity in gastric cancer cells through activation of hypoxia-inducible factor-1alpha. Oncol Rep 2012; 28: 2239-2246.
31. Zhou Z-L, Luo Z-G, Yu B, Jiang Y, Chen Y, Feng J-M et al. Increased accumulation of hypoxia-inducible factor- $1 \alpha$ with reduced transcriptional activity mediates the antitumor effect of triptolide. Mol Cancer 2010; 9: 268.

32. Wilczynski J, Duechler M, Czyz M. Targeting NF-kappaB and HIF-1 pathways for the treatment of cancer: part I. Arch Immunol Ther Exp (Warsz) 2011; 59: 289-299.

33. Wilczynski J, Duechler M, Czyz M. Targeting NF-kappaB and HIF-1 pathways for the treatment of cancer: part II. Arch Immunol Ther Exp (Warsz) 2011; 59: 301-307.

34. Matsunaga T, Imataki O, Torii E, Kameda T, Shide K, Shimoda H et al. Elevated HIF- $1 \alpha$ expression of acute myelogenous leukemia stem cells in the endosteal hypoxic zone may be a cause of minimal residual disease in bone marrow after chemotherapy. Leuk Res 2012; 36: e122-e124.

35. Dong-Feng Z, Ting L, Cheng C, Xi Z, Xue L, Xing-Hua C et al. Silencing HIF-1 $\alpha$ reduces the adhesion and secretion functions of acute leukemia hBMSCs. Braz J Med Biol Res 2012; 45: 906-912.

36. Tavor S, Petit I. Can inhibition of the SDF-1/CXCR4 axis eradicate acute leukemia? Semin Cancer Biol 2010; 20: 178-185.

37. Matsunaga T, Takemoto N, Sato T, Takimoto R, Tanaka I, Fujimi A et al. Interaction between leukemic-cell VLA-4 and stromal fibronectin is a decisive factor for minimal residual disease of acute myelogenous leukemia. Nat Med 2003; 9: 1158-1165.

38. Nervi B, Ramirez P, Rettig MP, Uy GL, Holt MS, Ritchey JK et al. Chemosensitization of acute myeloid leukemia (AML) following mobilization by the CXCR4 antagonist AMD3100. Blood 2009; 113: 6206-6214.

39. Reuter S, Gupta SC, Chaturvedi MM, Aggarwal BB. Oxidative stress, inflammation, and cancer: How are they linked? Free Rad Biol Med 2010; 49: 1603-1616.

40. Toyokuni S. Novel aspects of oxidative stress-associated carcinogenesis. Antioxid Redox Signal 2006; 8: 1373-1377.

41. Jin Y, Lu Z, Ding K, Li J, Du X, Chen C et al. Antineoplastic mechanisms of niclosamide in acute myelogenous leukemia stem cells: inactivation of the NF-kappaB pathway and generation of reactive oxygen species. Cancer Res 2010; 70: 2516-2527.

42. Guzman ML, Rossi RM, Karnischky L, Li X, Peterson DR, Howard DS et al. The sesquiterpene lactone parthenolide induces apoptosis of human acute myelogenous leukemia stem and progenitor cells. Blood 2005; 105: 4163-4169.

43. Baird L, Dinkova-Kostova AT. The cytoprotective role of the Keap1-Nrf2 pathway. Arch Toxicol 2011; 85: 241-272.

44. Rushworth SA, Bowles KM, MacEwan DJ. High basal nuclear levels of Nrf2 in acute myeloid leukemia reduces sensitivity to proteasome inhibitors. Cancer Res 2011; 71 1999-2009.

45. Yip NC, Fombon IS, Liu P, Brown S, Kannappan V, Armesilla AL et al. Disulfiram modulated ROS-MAPK and NFKB pathways and targeted breast cancer cells with cancer stem cell-like properties. Br J Cancer 2011; 104: 1564-1574.

(1)(2) Cell Death and Disease is an open-access journal published by Nature Publishing Group. This work is licensed under a Creative Commons Attribution-NonCommercialShareAlike 3.0 Unported License. To view a copy of this license, visit http://creativecommons.org/licenses/by-nc-sa/3.0/ 